\title{
EXPERIMENTAL DEVELOPMENT, TRADEOFF ANALYSIS AND DESIGN IMPLEMENTATION OF HIGH FORCE-TO-VOLUME DAMPING TECHNOLOGY
}

\author{
Geoffrey W. Rodgers ${ }^{1,2}$, J. Geoffrey Chase ${ }^{1}$, John B. Mander², \\ Nicholas C. Leach ${ }^{1}$ and Caleb S. Denmead ${ }^{1}$
}

\begin{abstract}
SUMMARY
Supplemental dampers are a means of repeatedly dissipating energy without damage to the underlying structure, increasing life-safety and helping provide better serviceability of structures following a major earthquake. High performance (small size) lead dampers are designed and tested to characterise their force-displacement behaviour and produce trade-off curves relating device geometry to force capacity, to parameterise the design space to enable further devices to be designed for structural applications. Peak forces of $120-350 \mathrm{kN}$ were obtained for devices that were all able to fit within standard structural connections.

Results show that prestressing the working material is critical to obtain optimal energy dissipation. Although previously characterised as extrusion dampers it is shown that classical extrusion modelling formulations do not strictly work well for this class of damper. Instead a coulomb type of stress-based model is proposed, with relationships presented that are independent of device scale. Empirical reduction factor equations are applied to the New Zealand Structural Design Actions to enable lead extrusion devices to be incorporated into structural design analyses. The overall results indicate that repeatable, optimal energy dissipation can be obtained in a compact device to minimise damage to critical buildings and infrastructure.
\end{abstract}

\section{INTRODUCTION}

Earthquakes and large environmental hazards cause significant structural damage, especially in structural connections. More specifically movement due to seismic loads causes major cracking and degradation in structural integrity. The associated damage can be difficult and expensive to repair even though modern structural design methods seek to localise damage to specific areas such as plastic hinge zones. Although this damage provides significant energy dissipation during a seismic event, it is preferable to dissipate energy without permanent structural damage.

The specific aim of this research is to further develop lead damping devices $[1,2]$ to absorb structural energy during an earthquake in a controlled manner, with repeatable behaviour. In contrast to the very large dampers previously deployed in New Zealand, the device must be sufficiently compact to allow placement in situations with tight volume constraints, while still providing the same high levels of force of the previous, much larger, devices. Ideally, the dampers should also be inexpensive and thus, commercially viable in regular use. This research uses lead as the working material due to its unique rheological properties and low re-crystallisation temperature. The high yield forces given by lead dampers give the ability to modify and reduce seismic response through hysteretic energy dissipation within the device alone, thus having the potential to markedly reduce damage in the structural elements.

Lead extrusion dampers have been used for energy dissipation in structural applications including base isolation [3]. Historically, these devices have been volumetrically very large, an impediment that has prevented their placement into applications with tight volume constraints, such as those shown in Figure 1. The large volume of previous devices also makes them relatively expensive to produce, limiting possible applications. This research modifies the basic designs and principles of these previous larger dampers to create significantly smaller $120-400 \quad \mathrm{kN}$ devices. Obtaining equivalent force levels in significantly smaller devices dramatically expands possible applications. Device lubrication was identified as a key factor in previous publications [3], but was not found to be necessary in this study.

One possible application for compact high force-to-volume extrusion dampers is in bridge piers, as shown schematically

\footnotetext{
${ }^{1}$ Department of Mechanical Engineering, University of Canterbury, Christchurch, New Zealand.

${ }^{2}$ Department of Civil Engineering, University of Canterbury, Christchurch
} 


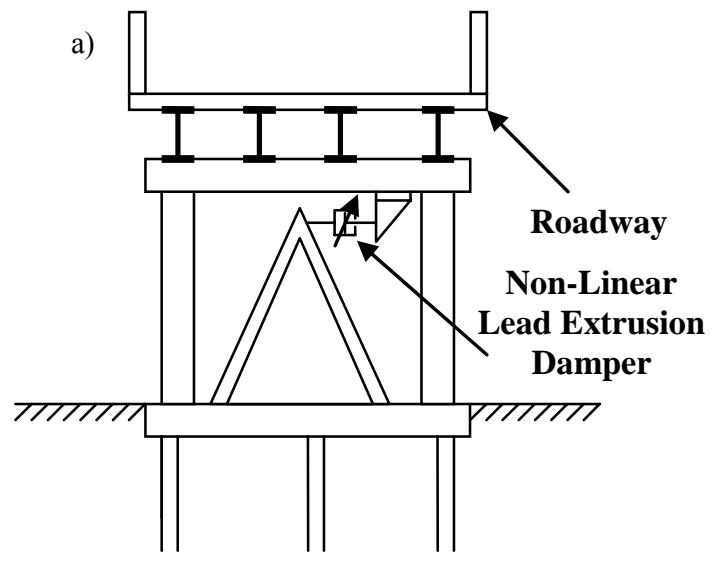

b)
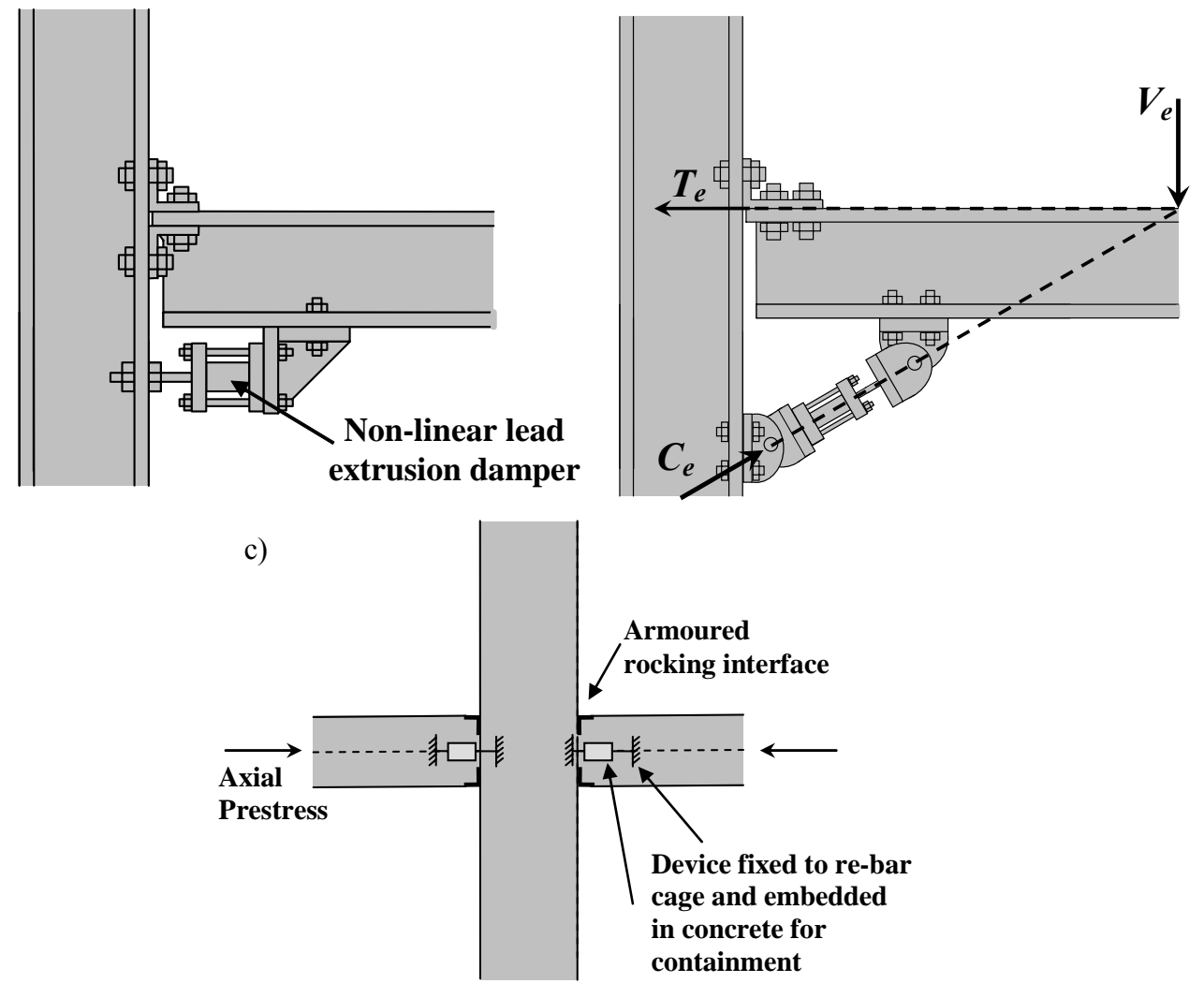

Figure 1: $\quad$ Possible applications for lead extrusions dampers; a) in a bridge pier, b) a seismic moment-frame steel beam-column connection, and a seismic load-balancing frame system, and c) in a reinforced concrete joint utilising Damage Avoidance Design (DAD)

in Figure 1a. In this application the load taken by the extrusion damper reduces the lateral demand on the columns. This method of using high force-to-volume extrusion dampers to provide supplemental damping is also very amenable to bridge piers that use Damage Avoidance Design (DAD). These piers typically have low inherent structural damping and are therefore particularly well augmented by the use of supplemental damping systems such as these high force-tovolume extrusion dampers. A pier utilising these principles can provide hysteretic energy absorption similar to that of a ductile concrete system, but do so in a damage-free manner, eliminating the need for costly repair following an earthquake.

Another possible application enabled by the reduced size of the device is presented in Figure $1 b$, where an extrusion damper is fitted directly into a steel beam-column connection. The extrusion damper provides a resistive force against joint rotation and consequently provides hysteretic energy absorption, preventing yielding of the elements of the main structural steel frame. The placement of the damper below the bottom flange of the beam results in larger displacements in the damper for a given design drift, and consequently larger energy dissipation. Figure $1 \mathrm{~b}$ shows both a seismic momentframe steel beam-column connection, and a seismic loadbalancing frame system, where the vertical component of the damper force provides a reaction to the shear force, $V_{\mathrm{e}}$.

Figure 1c shows a schematic representation of how a high force-to-volume extrusion based damper can be incorporated directly into a reinforced concrete bean-column connection that utilises Damage Avoidance Design (DAD) principles. The schematic of Figure 1c shows the extrusion damper cast directly into the end of the beam and connected to the column. In this application joint opening at the armoured rocking interface caused by joint rotation will displace the shaft of the damper, providing hysteretic energy absorption. The key advantage of a system such as this is the ability to provide hysteretic energy absorption in a damage-free and repeatable manner.

Extrusion dampers can be characterised into two groups based upon fundamental design differences. These are the bulgedshaft and constricted tube type extrusion dampers [3]. The relative merits of each type are documented [3] and focus upon ease of manufacture and ability to produce a repeatable 

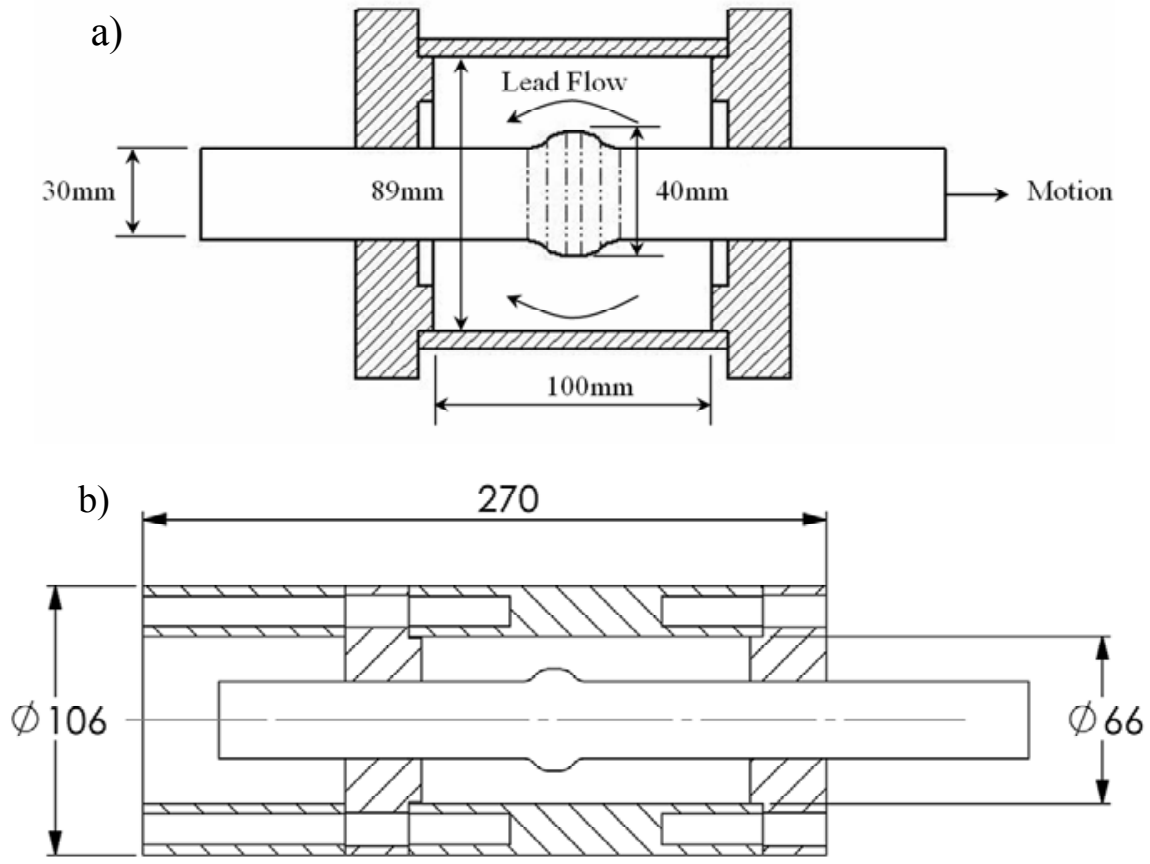

Figure 2: $\quad$ Cross-Sectional view of different lead extrusion damper configurations.

and consistent hysteresis loop. Both types of extrusion damper provide a resistive force by plastically extruding the working material through an orifice created by an annular restriction. Bulged-shaft extrusion dampers utilise a streamlined bulge on the central shaft to create the orifice, whereas the constricted tube type utilise a constriction on the bore of the outer cylinder. The bulged-shaft design was chosen for ease of manufacture and consequently low cost, an important consideration for widespread adoption of extrusion damping technology. A cross-sectional schematic of the bulged-shaft design is presented in Figure 2a.

The damper is designed to fit into confined spaces within and around structural connections, such as the applications presented in Figure 1. Figure 1a shows a damper set up to reduce lateral motion and absorb the response energy of a bridge deck and framing. In this application, simulations have shown that with a damper whose force capacity is rated around $10 \%$ of the weight of the structure, displacement reductions in excess of 50 percent can be expected [4]. The dampers could also be arranged longitudinally and anchored into bridge abutments to provide further protection if needed.

Figure $1 \mathrm{~b}$ shows a steel connection application with tight space constraints. Here, the damper must fit between the flanges of universal column sections nominally $350 \mathrm{~mm}$ deep (W14 in American steel design codes) to broaden possible application into steel high rise structures. As the joint rotates the clearance allows movement, transmitting the building motion to the damper, where resistive forces can be provided by the damper instead of through yielding of the structural steel elements. The shaft has been limited to approximately $30 \mathrm{~mm}$ in diameter, in-line with maximum fitting sizes commonly used in structural applications. Shaft material is limited to high strength steel, such as 4140 to eliminate manufacturing difficulties and cost increases associated less accessible materials. Figure $2 \mathrm{~b}$ shows the final prototype device design and dimensions with a $40 \mathrm{~mm}$ bulge on a 30 $\mathrm{mm}$ shaft. After a review of existing designs a tear-shaped bulge was chosen. The limiting factor in the design is the 30 $\mathrm{mm}$, high strength steel shaft with a yield force of approximately $500 \mathrm{kN}$, which is greater than the desired 250$400 \mathrm{kN}$ peak force levels for most structural applications.

This research reports on the testing and analysis of these extrusion based damping devices to obtain and characterise the desired behaviour and device design space. The primary goal is a device with a high force to volume ratio that enables widespread placement directly into structural connections. The majority of the tests undertaken were quasi-static squarewaves at approximately $0.1 \mathrm{~mm} / \mathrm{s}$. Varied speed tests were undertaken to characterise velocity dependence.

\section{METHODS}

\section{Basic Physics}

In a bulged-shaft lead extrusion damper, the lead is confined in a cylinder with the bulged-shaft through the centre, as shown in Figure 2. As the shaft is forced through the cylinder, the lead is forced to flow through the annular restriction. This plastic flow adsorbs a large amount of energy due to the shearing and deformation that occurs, providing high resistive forces. These high resistive forces enable an extrusion damper to be much stiffer, and capable of absorbing far more energy, than an equivalent sized fluid viscous damper. Two major factors limit the amount of energy that can be dissipated. First, the shaft yield load restricts force levels, and is defined by practical limits on shaft diameter and manufacturing and cost limitations on shaft material. Second, the heat produced by the damper on repeated cycles softens the surrounding lead and reduces resistance. Both factors can be reasonably managed by the device design.

One major issue with this method of damping is the formation of voids within the working material during extrusion. For a lead extrusion damper, this void formation can be attributed to the compression of the lead, expansion of the confining cylinder wall, and casting imperfections. These imperfections include air gaps or micro-sized voids that can be compressed. Hence, as the bulge moves through the material it is compressed into a smaller volume, leaving a trailing void. 


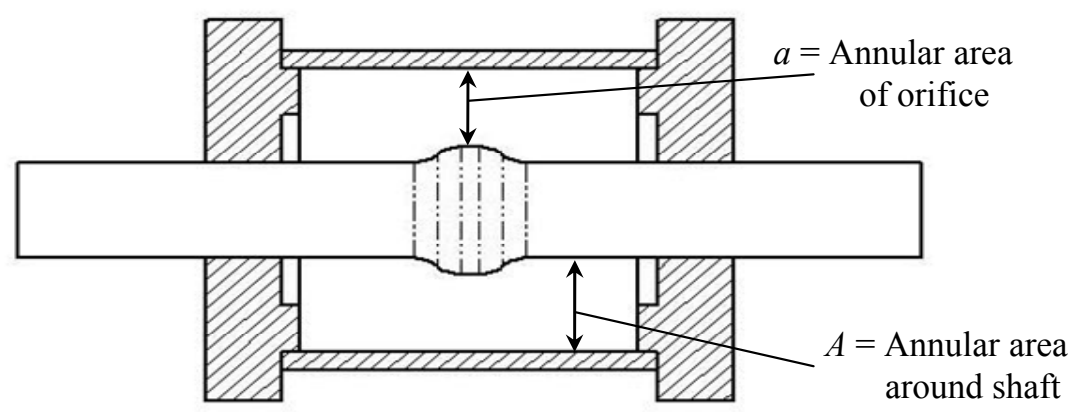

Figure 3: $\quad$ Schematic representation of the first device, showing area as used in Equation (1).

Thus, as the bulge passes through this void on subsequent cycles the damper experiences less resistance and dissipates much less energy. To minimize void formation the lead is prestressed, which helps reduce casting porosity and air gaps before the damper is used, and thus the size of the void, as a percentage of the total lead volume.

\section{Existing Models}

It appears that limited attempts have been made to fit a model to the experimental characteristics of similar dampers [5]. However, Pearsons et al [6] give a relationship between the force and associated cylinder and orifice areas during a similar extrusion process. As the lead in the damper is essentially being extruded between the bulge and cylinder of the damper this model was applied in an attempt to characterize the damper, and is defined:

$$
F=\left\langle\left[Y \ln \left(\frac{A}{a}\right)+Y\right] \exp (M)-Y\right\rangle(A-a)
$$

where $F=$ the extrusion force;

$Y=$ yield strength for the working material;

$A=$ annular area around the shaft as shown in

Figure 3, corresponding to the cross-sectional area of the extruding bar in Pearsons et al;

$a=$ annular area of orifice again shown in Figure 3, corresponding to the cross-section area of extruded rod in Pearsons et al.;

$(A-a)$ represents the projected face area of the bulge over which direct stress is applied to the shaft; and $M=$ a constant specific to the extrusion process, defined as:

$$
M=\frac{4 \mu L}{D}
$$

where $\quad \mu=$ the co-efficient of friction between the working material and steel shaft;

$L=$ the length of the shaft in sliding contact with the working material and device end caps, relating to the billet length in Pearson et al.; and

$D=$ Effective diameter corresponding to annular lead area $A$, as shown in Figure 3, corresponding to the billet diameter is Pearson et al.

Note that as the bulge diameter approaches the cylinder wall Equation (1) results in $F \rightarrow \infty$ as might be expected, where infinity implies plastic yielding of the shaft or bulge. It should be noted that the model presented in Pearson . is for a classic extrusion process and the model parameters do not translate directly to the parameters of the lead extrusion dampers, and close analogies are utilised to define the model.

\section{Experimental Methods}

Quasi-static compression tests were undertaken on an initial design, as shown in Figure 2a, to characterise the forcedisplacement behaviour. Further testing was undertaken at a range of speeds to experimentally determine the velocity exponent, which defines the increase in resistive force that is seen with increased shaft velocity as energy is dissipated more rapidly via faster extrusion. The velocity exponent can thus be utilised to relate the force from quasi-static test results to the force levels in full speed testing with velocities representing realistic values for the applications presented in Figure 1. This force-velocity relationship is defined [7]:

$$
F=C_{\alpha} \dot{x}^{\alpha}
$$

where $F=$ the extrusion damper force;

$$
\begin{aligned}
& \dot{x}=\text { velocity of the shaft; } \\
& \alpha=\text { velocity coefficient (constant); and } \\
& C_{\alpha}=\text { damper constant determined by physical } \\
& \text { prototype testing. }
\end{aligned}
$$

Testing and design was carried out using an iterative approach, with the results of each experimental test being used to calibrate empirical models and provide insight into the dominant factors of the extrusion process. The initial design utilised a conservative bulge diameter of $40 \mathrm{~mm}$ on a $30 \mathrm{~mm}$ shaft. This result, along with an estimate of force using Equation 1, led to the design and manufacture of a $50 \mathrm{~mm}$ diameter bulge on a $30 \mathrm{~mm}$ shaft. These results and their linear relationship were used to produce and test a $58 \mathrm{~mm}$ diameter bulge on a $30 \mathrm{~mm}$ diameter shaft, to provide more data and better characterise the empirical models.

Using these three results an accurate estimate of the forces that might be produced was obtained through interpolation between experimental results. Further testing with the 40 and $50 \mathrm{~mm}$ bulges on a device with a $66 \mathrm{~mm}$ internal cylinder diameter, as shown in Figure 2b, added more data with respect to variation in cylinder size and volume. The overall test results were compared to Equation (1) to create empirical design trade-off curves. The effect of prestressing the cast lead was also investigated. The devices tested comprise:

- 1a) Device of Figure 2a with $40 \mathrm{~mm}$ and $50 \mathrm{~mm}$ bulges without prestress

- 1 b) Device of Figure 2a with $40 \mathrm{~mm}, 50 \mathrm{~mm}$ and 58 $\mathrm{mm}$ bulges and prestress

- 2) Device of Figure $2 \mathrm{~b}$ with $40 \mathrm{~mm}$ and $50 \mathrm{~mm}$ bulges and prestress

Devices $1 \mathrm{a}$ and $1 \mathrm{~b}$ are used to examine the effect of prestressing the lead and the corresponding effect on the peak force achieved, as well as the shape of the hysteresis loop, and ability to achieve repeatable device behaviour. Devices $1 \mathrm{~b}$ and 2 provide a variety of results with prestress, with the design 
a)
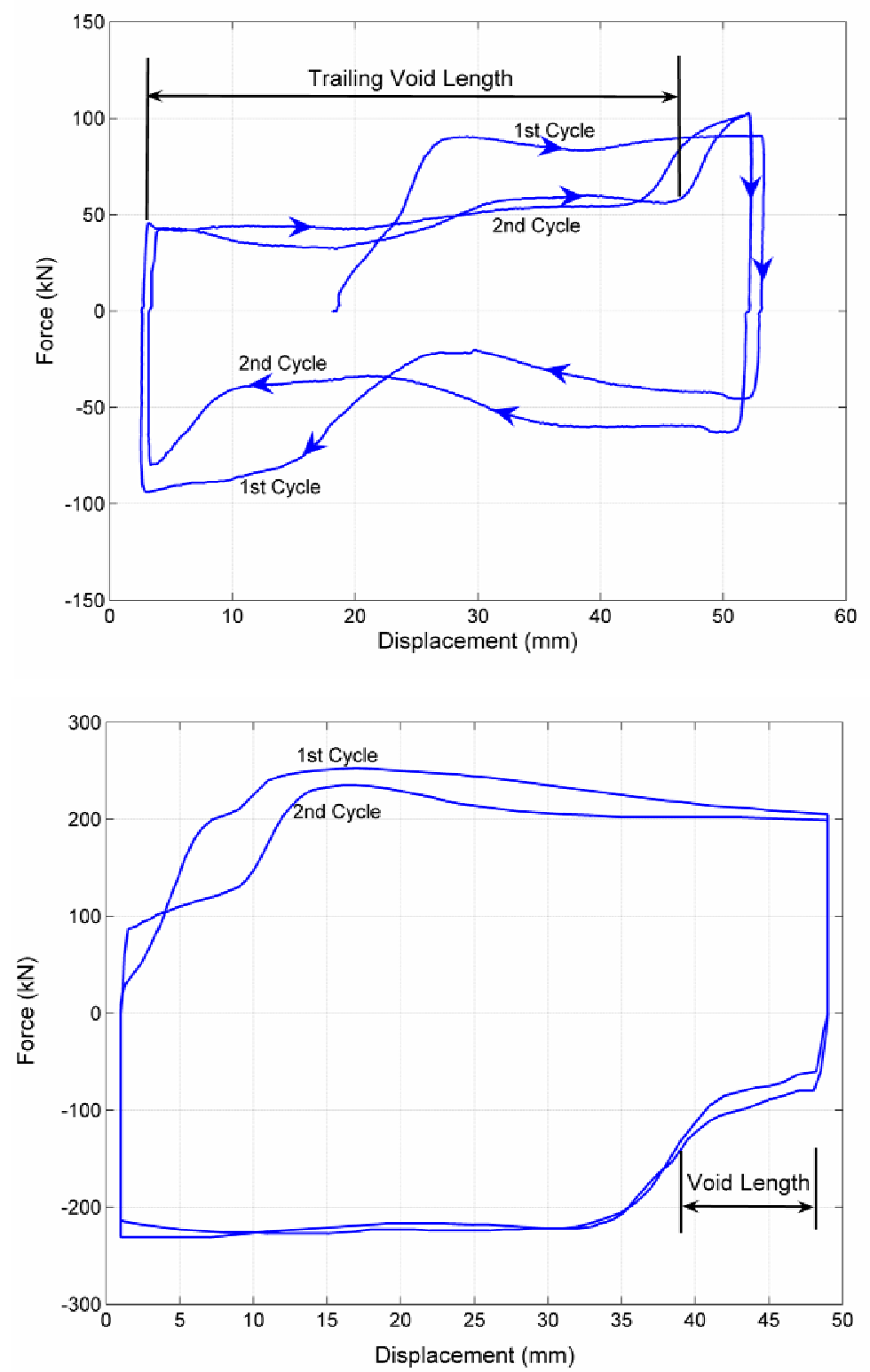

Figure 4: Hysteresis loop for Device 1 with a) $40 \mathrm{~mm}$ diameter bulge and b) $50 \mathrm{~mm}$ diameter bulge

parameters of cylinder diameter and bulge size being independently varied.

\section{RESULTS AND DISCUSSION}

\section{Results without prestress}

Initial manufacturing methods used as-cast lead as the working material. When the lead solidified, shrinkage of the working material occurred along with the development of small micro-voids due to trapped air. Hence, the lead did not completely fill the volume inside the damper, and these extra volumes can be compressed and contribute to creating a trailing void behind the bulge. The presence of trapped air was amplified by the rapid solidification of the lead as it contacted the cylinder walls and endcap. To mitigate this problem, the devices were preheated to reduce the cooling rate and enable the trapped air to escape before the lead solidifed.

The hysteresis loop for a $40 \mathrm{~mm}$ diameter bulge and no prestress, presented in Figure $4 \mathrm{a}$, shows the first stroke produced a peak force of $90 \mathrm{kN}$ reasonably constant throughout the stroke. During the return stroke the force was significantly lower at around $50 \mathrm{kN}$ until "new" material was enetered at the device midpoint. After the initial return stoke the peak force was effectively constant at around $50 \mathrm{kN}$, therefore reducing the area enclosed within the hysteresis loop and reducing the energy absorbed by the damper. An important observation is that the $90 \mathrm{kN}$ force produced on the first stroke was nearer the value predicted using Equation (1).

The cause of this drop in force is the coring out of the lead as the bulge travels through the cylinder. This coring effect is facilitated by the lead compressing and closing up micro-voids and casting porosity, rather than flowing around the bulge as desired. The cylindrical trailing void produced had a measured diameter of $38 \mathrm{~mm}$, indicating that the lead was only flowing back about $1 \mathrm{~mm}$ around each side. This cylindrical coring effect results in much less resistance on the bulge as it is forced through the cylinder, reducing the resistive force produced. Effectively, the lead compressed enough so that approximately $8-10 \%$ of its volume was lost. This reduction in volume, coupled with the $40 \mathrm{~mm}$ bulge made a relatively constant void of $38 \mathrm{~mm}$ diameter along the entire shaft length.

The hysteresis loop for the $50 \mathrm{~mm}$ bulge without prestress shows similar characteristics to the $40 \mathrm{~mm}$ case in Figure 4a, but with reduced coring effects. Because of the increase in diameter of the bulge the same volume of air void, as a 


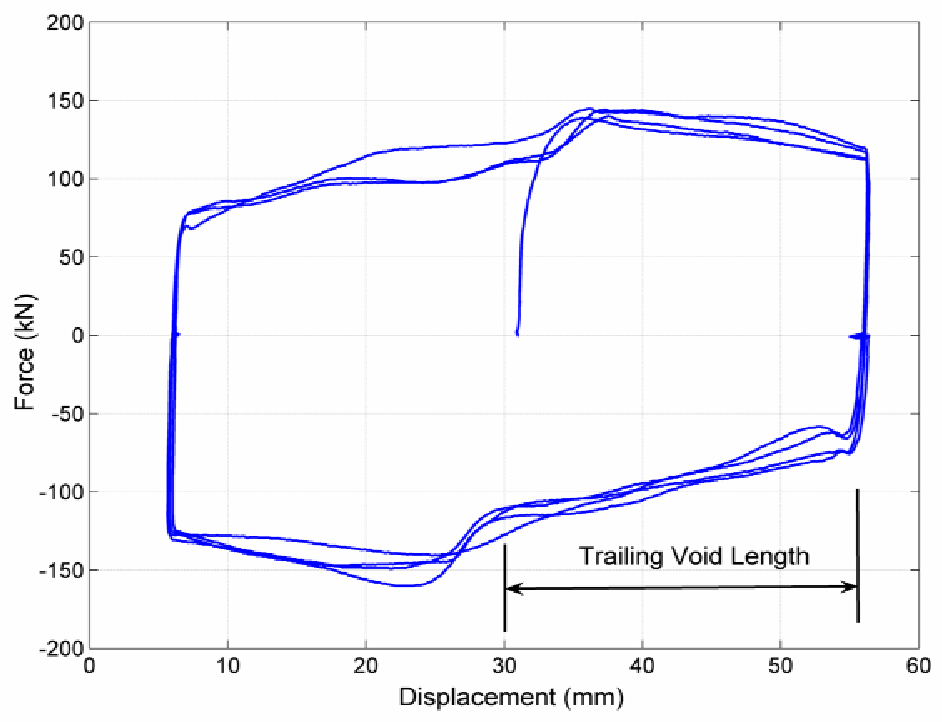

Figure 5: $\quad$ Hysteresis Loop for prestressed lead and 40mm diameter bulge.

percentage of total volume, has a reduced length, as seen in Figure $4 \mathrm{~b}$. These results thus show a more optimal 'square' loop than the $40 \mathrm{~mm}$ case, with small "cut-outs" from the corners of the hysteresis loop being the only effects of the trailing void. After this initial cut-out, the peak force from the original stroke is reached, enabling the damper to absorber nearly as much energy as on the initial stroke. The shape of the loop shows the trailing void created behind the bulge to be only approximately $10 \mathrm{~mm}$, indicating a much improved result. The peak force produced was between $220 \mathrm{kN}$ and 230 $\mathrm{kN}$, and closer to expected values.

The coring effects may be amplified if an insufficient confining force is provided by the outer cylinder. Strain gauges mounted on the $6.5 \mathrm{~mm}$ thick cylinder wall of Device 1 revealed plastic deformation of up to $2 \%$ as the bulge passed. This deformation would also contribute to the formation of the trailing void as perfect confinement of the working material was not maintained. Reducing the void is important to increase energy dissipation by maximizing time at peak force. In an attempt to minimise the amount of compression of the lead during cyclic testing and consequently reduce the trailing void formation the same devices were tested after first pre-stressing the as-cast lead.

\section{Results with Prestress}

Following solidification of the as-cast lead within the cylinder, a $400 \mathrm{kN}$ force was applied to compress any air voids present in the casting and put a residual compressive stress on the material. Figures $5,6 \mathrm{a}$, and $6 \mathrm{~b}$ show the hysteresis loops of the results from Device 1(b) with 40, 50 and $58 \mathrm{~mm}$ bulges respectively. Figures 7a-b shows the hysteresis loops of the results from Device 2 with 40 and $50 \mathrm{~mm}$ bulges. Due to slight variability of the peak force during any single stroke of the device, no single value can accurately represent force levels. Therefore, both the peak force, and an estimated average force level across the post-yield portion of the loop are included to indicate hysteretic performance of the devices. Table 1 summarises these peak and average forces for all five sets of results, where the average force is the effective mean value across the top, peak force portion of the hysteresis loop.
Table 1. Summary of prestressed device results

\begin{tabular}{ccc}
$\begin{array}{c}\text { Bulge Size } \\
(\mathbf{m m})\end{array}$ & $\begin{array}{c}\text { Max Force } \\
(\mathbf{k N})\end{array}$ & $\begin{array}{c}\text { Average } \\
\text { Force }(\mathbf{k N})\end{array}$ \\
\hline Device 1b) & & \\
40 & 170 & 125 \\
50 & 255 & 230 \\
58 & 360 & 305 \\
Device 2 & & \\
40 & 205 & 160 \\
50 & 350 & 300 \\
\hline
\end{tabular}

The hysteresis loops presented in Figures 5-6 are much 'squarer', more optimal loops, showing far less influence due to void formation. The trailing void remains, but has been reduced dramatically by the application of prestress. The results presented in Figure 6 show that trailing voids are only approximately $2-3 \mathrm{~mm}$ long for the larger $50 \mathrm{~mm}$ bulge compared with $20 \mathrm{~mm}$ for the $40 \mathrm{~mm}$ bulge as presented in Figure 5. Again a fixed void volume, as a percentage of total lead volume, represents a shorter trailing void for the larger diameter bulges. However, these voids are at least 3 times smaller in volume than without prestress, resulting in much smaller drops in force, more time at peak force, and consequently more energy absorption and dissipation.

Another important aspect of the prestress is the rise in force produced by the dampers. More specifically, there is an increase of $80 \mathrm{kN}$ for the $40 \mathrm{~mm}$ bulge and $35 \mathrm{kN}$ for the $50 \mathrm{~mm}$ bulge from the non-prestressed results of Device 1a. This result can be attributed to the reduction in casting porosity by the compression of the lead in the prestressing process, and greater constriction of the lead due to the residual compressive stress. However, as the larger bulge size also provides a greater constriction, as the bulge size increases this difference in force decreases.

Prestressing decreases the trailing void volume by 3-5 times when compared to non-prestressed tests. This reduction was determined by post-testing disassembly and measurement of the internal voids within the devices, and can also be seen in the hysteresis loops. A force reduction still remains over part of the stroke when the smaller $40 \mathrm{~mm}$ bulge is used, and this 
a)

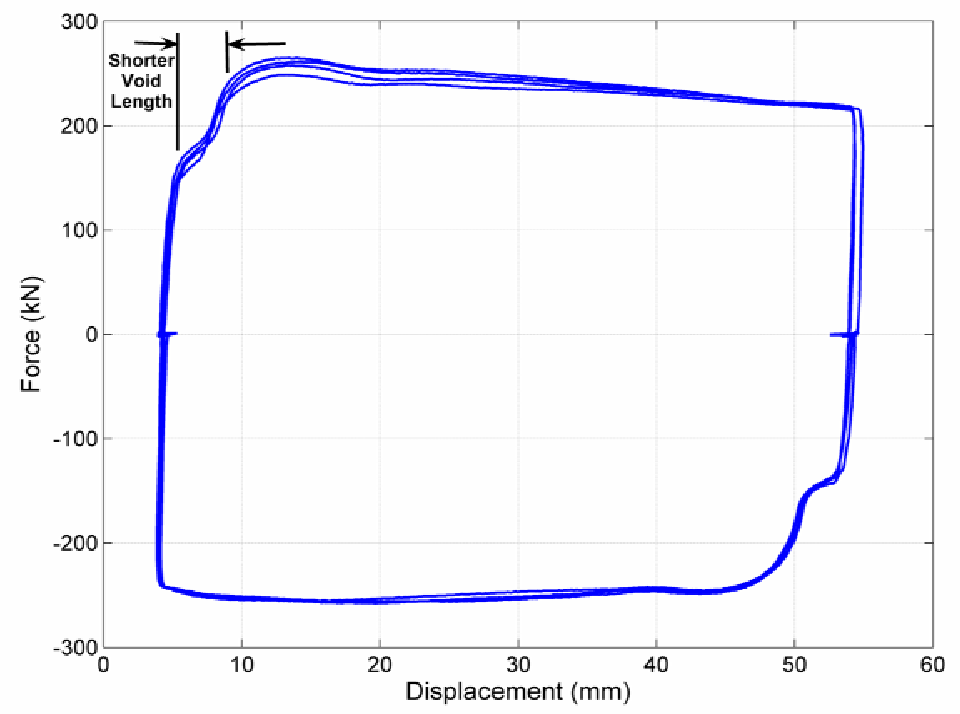

b)

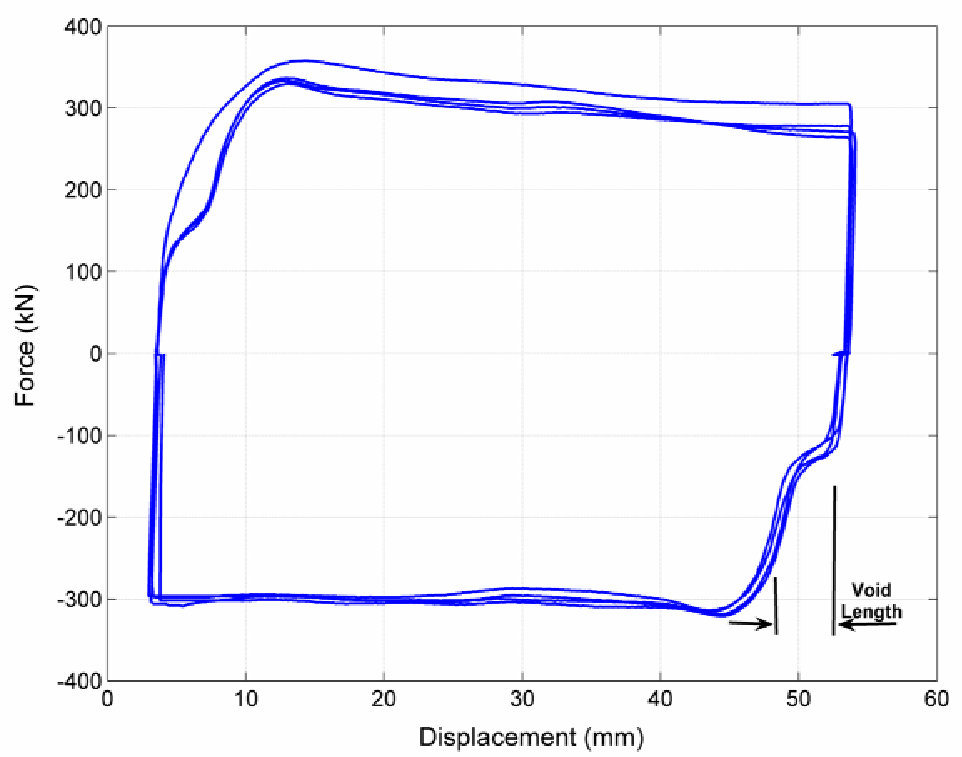

Figure 6:

Hysteresis loop for prestressed lead and a) a $50 \mathrm{~mm}$ diameter bulge, and b) a $58 \mathrm{~mm}$ diameter bulge.

effect appears unavoidable unless an extremely large prestress force is applied. In this case, the force reduction is also partly due to the relatively low height of the $40 \mathrm{~mm}$ bulge over the $30 \mathrm{~mm}$ shaft. Since the $40 \mathrm{~mm}$ bulge has a small face area, a given trailing void volume will produce a noticeable reduction in force over a longer part of the stroke. This effect is minimised using the larger bulge sizes of 50 and $58 \mathrm{~mm}$.

The results of device $1 \mathrm{~b}$ with the larger 50 and $58 \mathrm{~mm}$ diameter bulges, presented in Figure 6, show almost no reduction in force due to void formation. The only influence of any coring effects is a small 'cut out' in the hysteresis loop for the first $4 \mathrm{~mm}$ of every stroke, but this small reduction is not large enough to have significant effects. This cut out may be reduced further by applying greater pre-stress load to the lead. The almost perfectly square shape of these results means the device is absorbing almost the maximum amount of energy per cycle within the given force and displacement constraints.

Similar results are presented in Figure 7 for Device 2, with 40 and $50 \mathrm{~mm}$ diameter bulges, although these loops show slightly greater coring effects. It is important to note that the design of Device 2 permitted limited prestress of only around $100 \mathrm{kN}$. Device 2 still achieved average forces of $160-300 \mathrm{kN}$ and relatively square hysteresis loops. Most of the force increase compared to Device $1 \mathrm{~b}$ is attributed to the smaller internal cylinder diameter of $66 \mathrm{~mm}$ compared to $89 \mathrm{~mm}$ for Device 1b, although Device 2 also had a significantly larger cylinder wall thickness than that of device 1 , providing better containment.

The main aspect of this research has focused on enabling dampers to be constructed that can fit directly into structural connections. Two sets of dampers have been constructed for use in reinforced concrete beam-column connections that utilise an emerging Damage Avoidance Design (DAD) philosophy. As discussed earlier, these dampers are particularly well suited to joints that utilise DAD as the joint opening that occurs during the rigid body component of the total joint drift displaces the damper shaft and provides the desired energy absorption.

The first set of dampers to be placed into a joint had a design force of $120 \mathrm{kN}$, and was designed to be externally mounted to the joint, through a steel plate cast into the beam. To achieve the desired design force of $120 \mathrm{kN}$, a different scale of device to that previously constructed was required. All previous devices constructed had utilised a $30 \mathrm{~mm}$ nominal shaft diameter. The requirement of a smaller device, and the lower design force lead to a design using a $20 \mathrm{~mm}$ nominal shaft diameter, $32 \mathrm{~mm}$ bulge diameter, and internal cylinder diameter of $50 \mathrm{~mm}$. The hysteresis loops for one of these 
a)

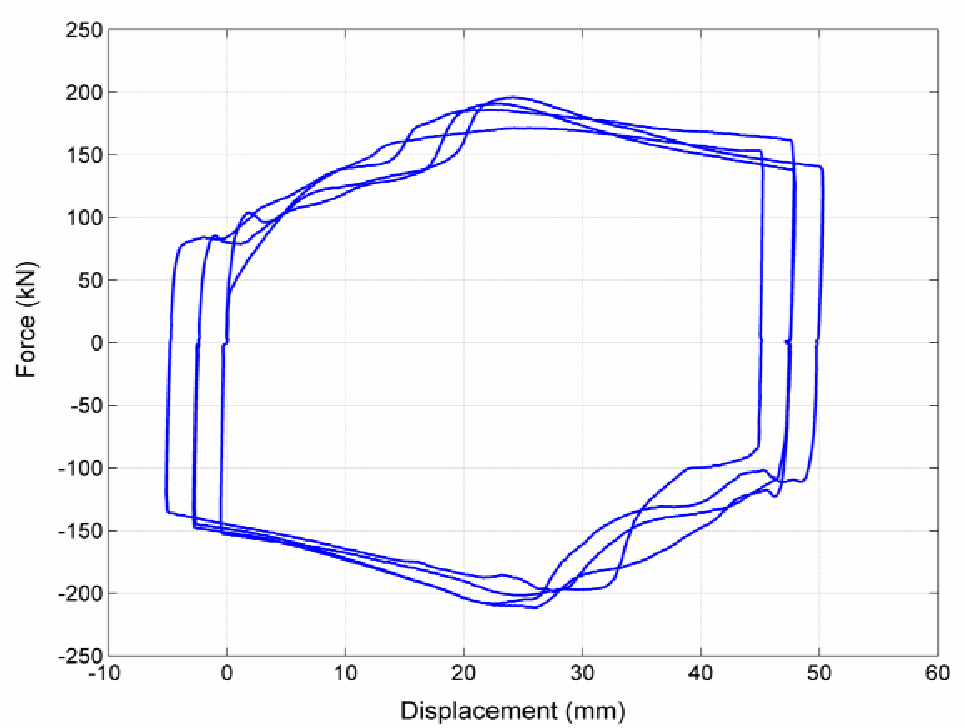

b)

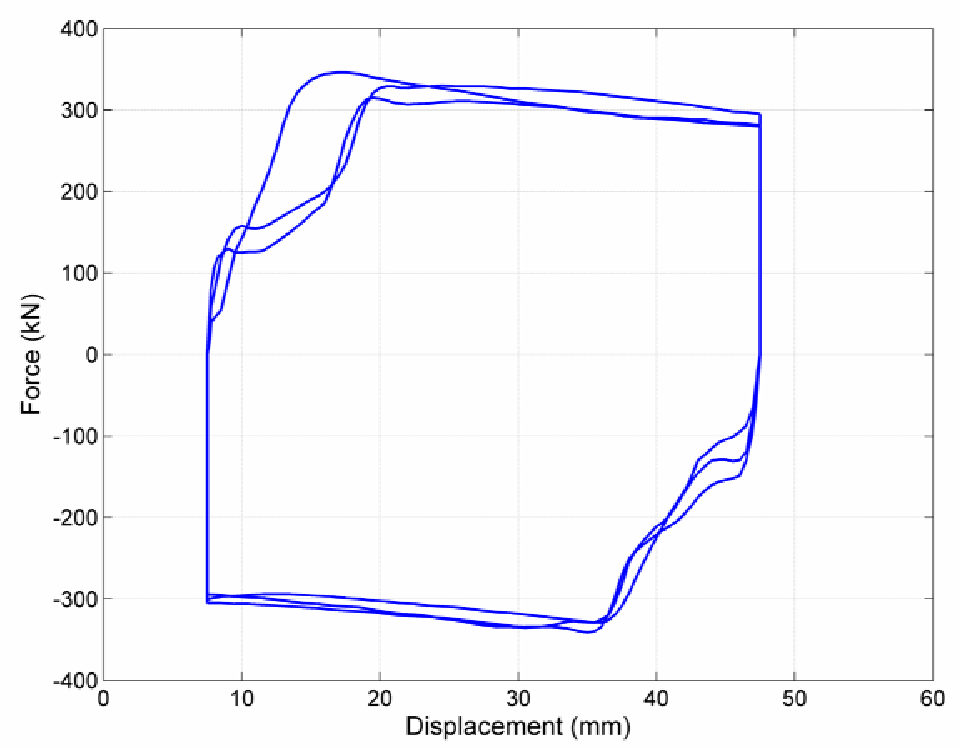

Figure 7:

Hvsteresis loop for device 2 with prestressed lead, and a) $40 \mathrm{~mm}$, and b) $50 \mathrm{~mm}$ diameter bulges.

devices subjected to quasi-static testing is presented in Figure $8 \mathrm{a}$, along with a photograph of the device with a standard 355 $\mathrm{ml}$ soft drink can to indicate scale presented in Figure $8 \mathrm{~b}$. Finally, in Figure 8c is a photograph of the damper mounted to the reinforced concrete beam-column connection that utilises DAD, closely similar to the schematic presented in Figure 1c.

Heating of the dampers as energy is dissipated is an important consideration as the smaller dampers provide a smaller heat sink for the heat produced. Cyclic testing was performed at velocities of $16-20 \mathrm{~mm} / \mathrm{sec}$, which represents realistic earthquake speeds for joint rotations. During these cyclic testing the force level dropped by $10-20 \%$, but tended to reach a steady state without any further reductions. This force reduction is unlikely to pose any difficulties for earthquake response reduction, as there will usually only be 3-4 large pulses during an earthquake and heat build-up will not measurably impact behaviour.

\section{Experimental Relationships}

The average peak forces from the test results, presented in Table 1, were used to relate device parameters to force produced. Figure 9a shows the relationship of bulge diameter to force for the experimental results, and includes the estimated $40 \mathrm{kN}$ friction force, with the upper force limit defined by shaft yield strength at maximum bulge diameter for
Device 1b. Also plotted in Figure 9a is the relationship between Force and Bulge diameter as predicted by the extrusion model of Equation (1). Note that the fitted line is almost exactly linear and thus different from the extrusionbased behaviour expected from Equation (1).

Figure $9 \mathrm{~b}$ shows the relationship between force and the device area ratio. This area ratio is defined as the net projected face area of the bulge divided by the net lead area. The net projected face area of the bulge is defined as the crosssectional area of the bulge with the cross-sectional area of the shaft subtracted. The net lead area is the total internal cylinder area, with the shaft cross-sectional area subtracted to give the net annular cross-sectional area of lead that is present around the shaft. The overall area ratio, as plotted on the horizontal axis of Figure $9 \mathrm{~b}$ is a measure of the area reduction required for extrusion, ranging from $[0,1]$. When plotting all three devices that have included prestress in Figure 9b, the force is seen to be a linear relationship to the area ratio for each device, with each device exhibiting a different linear relationship. The linear trends show an increasing level of force as the internal cylinder diameter is increased, clearly indicating that, as expected, the scale of the device affects the force level.

In the interests of enabling future designs to incorporate devices of different scales, it is desirable to determine experimental relationships that are independent of the device 
a)

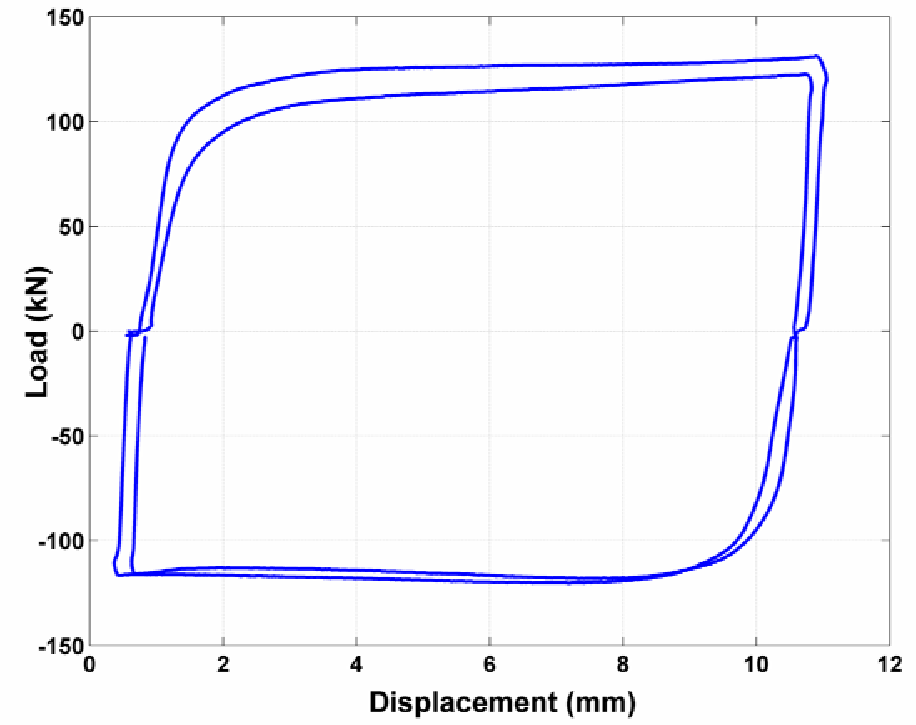

b)

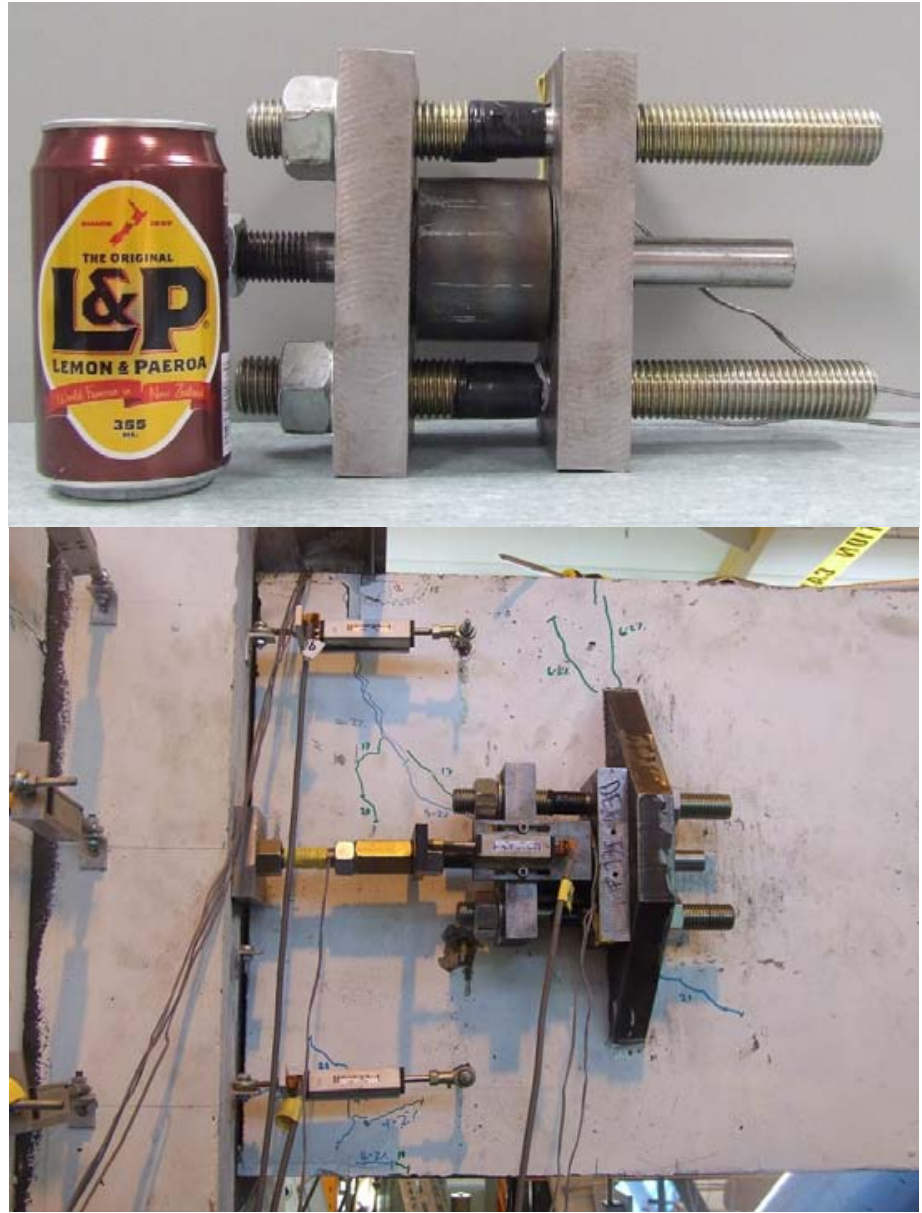

Figure 8:

High force-to-volume extrusion damper for placement onto reinforced concrete joint - a) hysteresis loop of damper, b) damper photograph with $355 \mathrm{ml}$ soft drink can for scale, and c) photograph of the damper mounted externally onto the joint.

scale. Therefore, a normalisation procedure is required to remove the scale factor, and for convenience the internal cylinder diameter is chosen as this scale parameter. After this normalisation procedure, the normalised force level for each device is obtained, and represents the average experimental force level divided by the internal cylinder diameter. Figure 9c presents the normalised force level plotted against the area ratio, and shows a relatively strong linear relationship for all experimental results independent of device scale. The development of an overall estimate of device force independent of device scale enables future devices of varying scale to be designed quickly and accurately and is an important step to provide a basis for eventual uptake by the profession.

As an alternative to the extrusion theory presented, a more straight forward stress-based model was considered. Using the Mohr-Coulomb failure criteria for a quasi-brittle material, the resistive force for the device, $D_{D}$, is given as:

$$
D_{D}=\tau_{D} A_{\text {shaft }}+\sigma_{o} A_{b u \lg e}
$$

where $\tau_{D}=$ shear stress due to the load between the shaft and the lead;

$\sigma_{o}=$ direct stress imposed on the bulge face area, 
a)

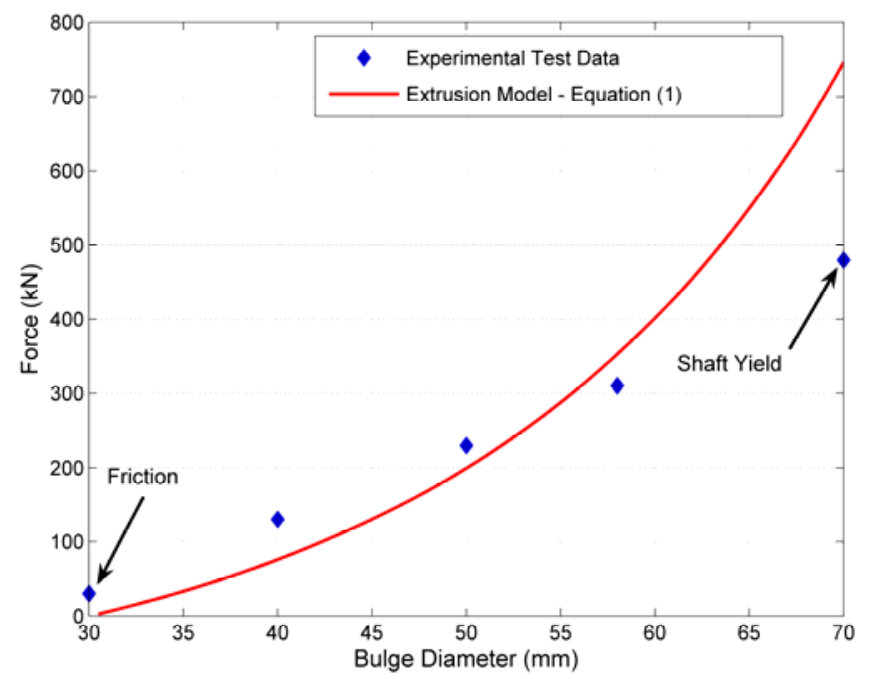

b)

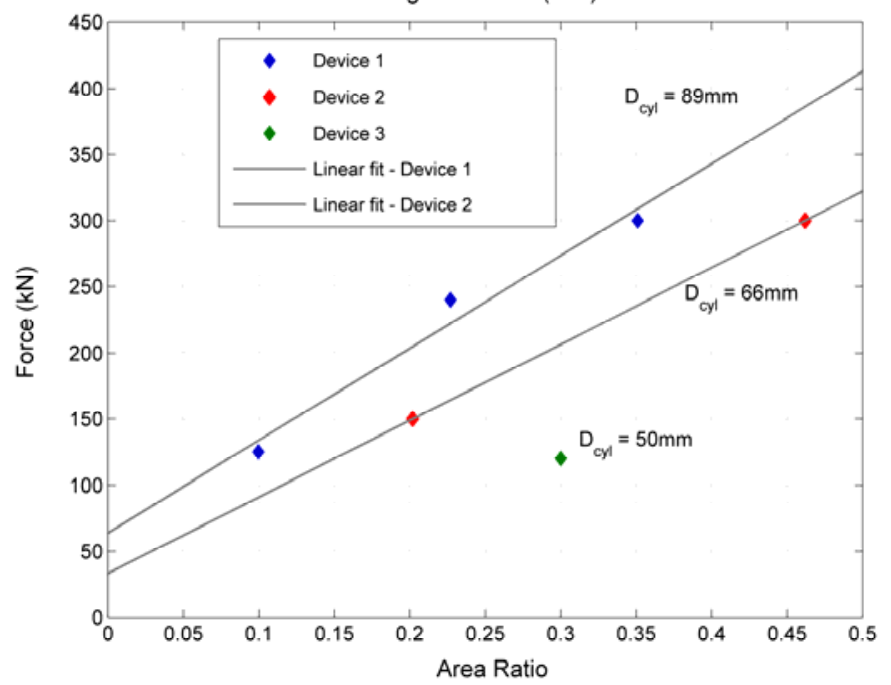

c)

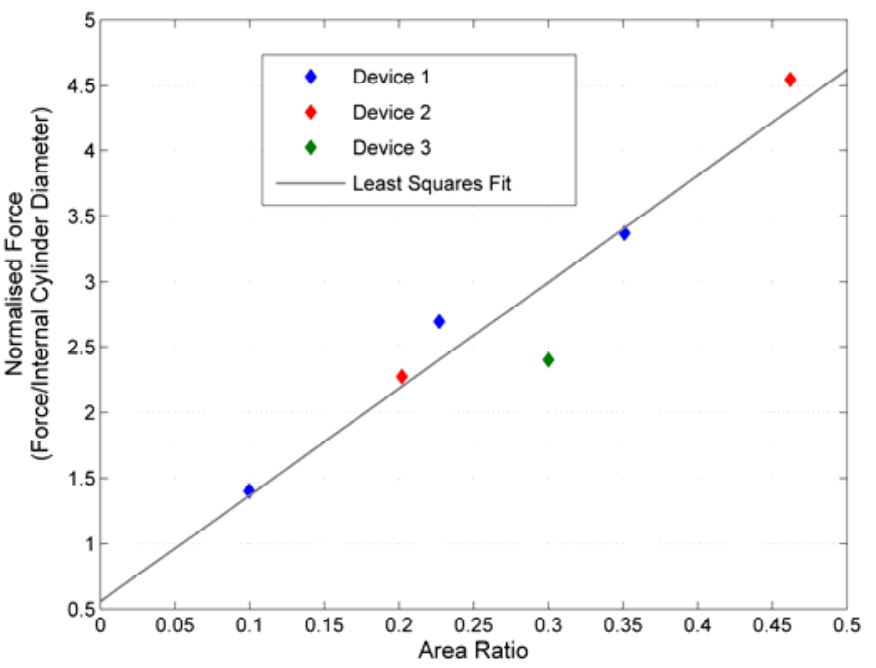

Figure 9:

a) Relationship of force to bulge diameter for Device 1 b experimental versus Equation (1); b) Force vs Area Ratio for all devices; and c) Normalised Force vs Area Ratio for all devices.

$$
\begin{aligned}
& A_{\text {shaft }}=\text { surface area of the shaft; and } \\
& A_{\text {bulge }}=\text { annular area of the bulge. }
\end{aligned}
$$

Fitting the values of $\tau_{D}$ and $\sigma_{o}$ to the experimental data of Device $1 \mathrm{~b}$ and using a least squares fit yielded $\tau_{D}=4.0 \mathrm{MPa}$ and $\sigma_{o}=140 \mathrm{MPa}$.

These results can be found from examining Mohr's circle of stress for lead in the unconfined condition. First, the maximum shear stress is $\tau_{D}=\sigma_{\mathrm{y}} / 2$ where $\sigma_{\mathrm{y}}=$ unconfined yield stress of lead, equal to 6-11 $\mathrm{MPa}$. Second, $\sigma_{o}$ is well above the unconfined compression strength. However, further insight can be obtained by accounting for the confining stress applied to the lead by the cylinder. Due to the thin wall thickness of the cylinder in Device 1b the maximum confining stress can be defined as a value corresponding to the yield stress of the cylinder wall. Basic thin-walled pressure vessel theory gives a value of the confining stress applied to the lead, $\sigma_{C}$, equal to $36 \mathrm{MPa}$ at an induced stress of $250 \mathrm{MPa}$ in the mild steel 

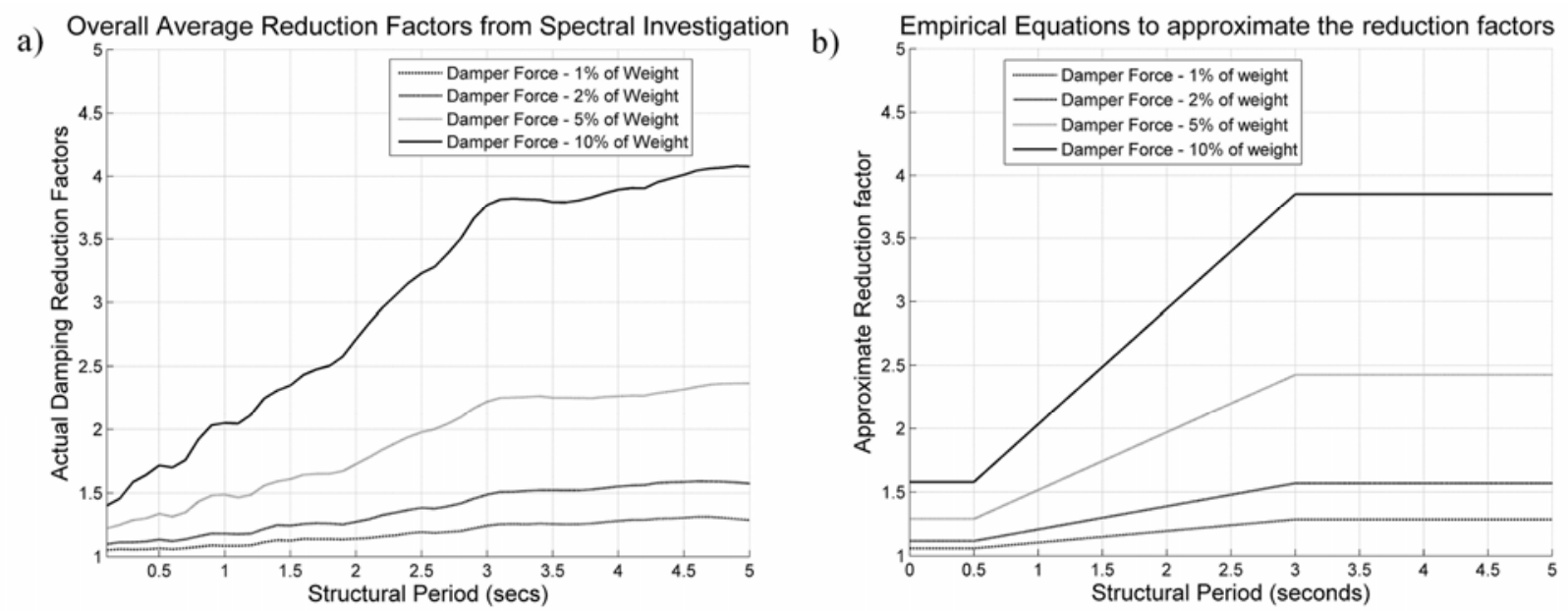

Figure 10: a) Overall average reduction factors from spectral investigation, and b) the multiple equation model to approximate damping reduction factors for design purposes.

cylinder. This result implies a confined lead strength relationship defined as:

$$
\sigma_{P b C}=\sigma_{y}+3.6 \sigma_{C}
$$

where $\quad \sigma_{P b C}$ is the confined yield stress of the lead.

The shear stress calculated, leads to a force for an un-bulged shaft of $37.7 \mathrm{kN}$ which is close to experimental estimates of the friction force from un-prestressed experiments with $40 \mathrm{~mm}$ bulge. However, more results are needed to fully verify this new model.

\section{Applications for Design Implementation in New Zealand Conditions}

An important consideration when designing and analysing structural damping devices is providing a platform for uptake by the profession. A key aspect to providing this platform is through methods that relate the hysteretic damping provided by these devices to known design guidelines. Previous work in this field includes Skinner et al. [5], and an earlier paper presented empirical equations to model the damping reduction factors that can be obtained when lead extrusion dampers are added to a structure in a spectral analysis [4]:

$$
\begin{array}{lc}
B_{a}=1+5.8 \varepsilon & (T<0.5 \mathrm{sec}) \\
B_{d}=1+27.6 \varepsilon & (T \geq 3 \mathrm{sec}) \\
B_{v}=1+(8.72 T+1.44) \varepsilon & (0.5 \leq T<3 \mathrm{sec})
\end{array}
$$

where $\varepsilon=$ the non-dimensional damper capacity, defined as the damper force at a standard reference velocity of $1 \mathrm{~m} / \mathrm{s}$, divided by the seismic weight of the structure; $T=$ the natural period of vibration of the structure.

These damping reduction factors are based upon a multiple equation model and segregate the spectra into three regions based upon existing bifurcation points. Plots of the overall average reduction factors from the spectral investigation performed in Rodgers et al. [4] are presented in Figure 10a, alongside a plot of the multiple equation model in Figure 10b. This figure shows that the multiple equation model provides a suitable estimate of the average reduction factors across the entire spectrum.

The earlier paper utilised these reduction factors and presented design implementation equations based upon the capacity spectrum method using customary US practice [4]. To make the design implementation more applicable to the New Zealand profession, reduction factors and design implementation are now presented to conform to the New Zealand Structural Design Actions, 1170.5. The capacitydemand spectrum is created using spectral shape factors taken from NZS 1170.5 [8]. The base-shear coefficient with respect to the peak ground acceleration is taken as the lesser of:

$$
\begin{aligned}
& C_{h}(T)=1.0+1.35(T / 0.1) \\
& C_{h}(T)=2.35 \\
& C_{h}(T)=1.60(0.5 / T)^{0.75} \\
& C_{h}(T)=1.05 / T \\
& C_{h}(T)=3.15 / T^{2}
\end{aligned}
$$

For implementation it is necessary to remove the structural period, $T$, from the equations. Thus, using the equation for the natural period, and substituting $W=m g$ and $K=F_{y} / \Delta_{y}$ into the equation and rearranging gives:

$$
T=2 \pi \sqrt{\frac{m}{K}}=2 \pi \sqrt{\frac{W \Delta_{y}}{g F_{y}}}
$$

where $\quad T=$ the natural period of vibration of the structure;

$m=$ mass of the structure;

$K=$ structural stiffness;

$W=$ structural weight;

$\Delta_{y}=$ structure yield displacement;

$g=$ acceleration due to gravity; and

$F_{y}=$ structure yield force;

For convenience, the normalised base shear capacity can be defined as $C_{c}=F_{\mathrm{y}} / W$. This can be substituted into Equation (10) to give:

$$
T=2 \pi \sqrt{\frac{\Delta_{y}}{C_{c} g}}
$$

Furthermore, Equation (11) can now be substituted into Equations (9i-v) to obtain spectral shape factors that are independent of structural period, $T$ :

$$
\begin{aligned}
& C_{h}=1.0+84.8 \sqrt{\frac{\Delta_{y}}{C_{c} g}} \\
& C_{h}=2.35 \\
& C_{h}=0.24\left(\frac{C_{c} g}{\Delta_{Y}}\right)^{0.375}
\end{aligned}
$$




$$
\begin{aligned}
& C_{h}=0.167 \sqrt{\frac{C_{c} g}{\Delta_{y}}} \\
& C_{h}=0.08 \frac{C_{c} g}{\Delta_{y}}
\end{aligned}
$$

The overall Design Response Spectrum, $C(T)$, can be expressed as the product of the spectral shape factor, $C_{h}(T)$, the hazard factor, $Z$, the return period factor, $R$, and the nearfault factor, $N(T, D)$ :

$$
C(T)=C_{h}(T) Z R N(T, D)
$$

When the added damping effects are taken into consideration, through the use of the damping reduction factors given in
Equations (6-8):

$$
C(T)=\frac{C_{h}(T) Z R N(T, D)}{B_{\varepsilon}}
$$

Moreover, by neglecting near-field effects $(N=1)$, and substituting out the period, the following equation can be obtained:

$$
C_{d}=C_{h} \frac{Z R}{B_{\varepsilon}}
$$

where $\quad C_{d}$ is defined as the base shear demand.

If the normalised base-shear capacity is set to be equal to the demand in Equation (15), ie: $C_{c}=C_{d}$, and then re-arranged, the seismic performance can be expressed as:

a)
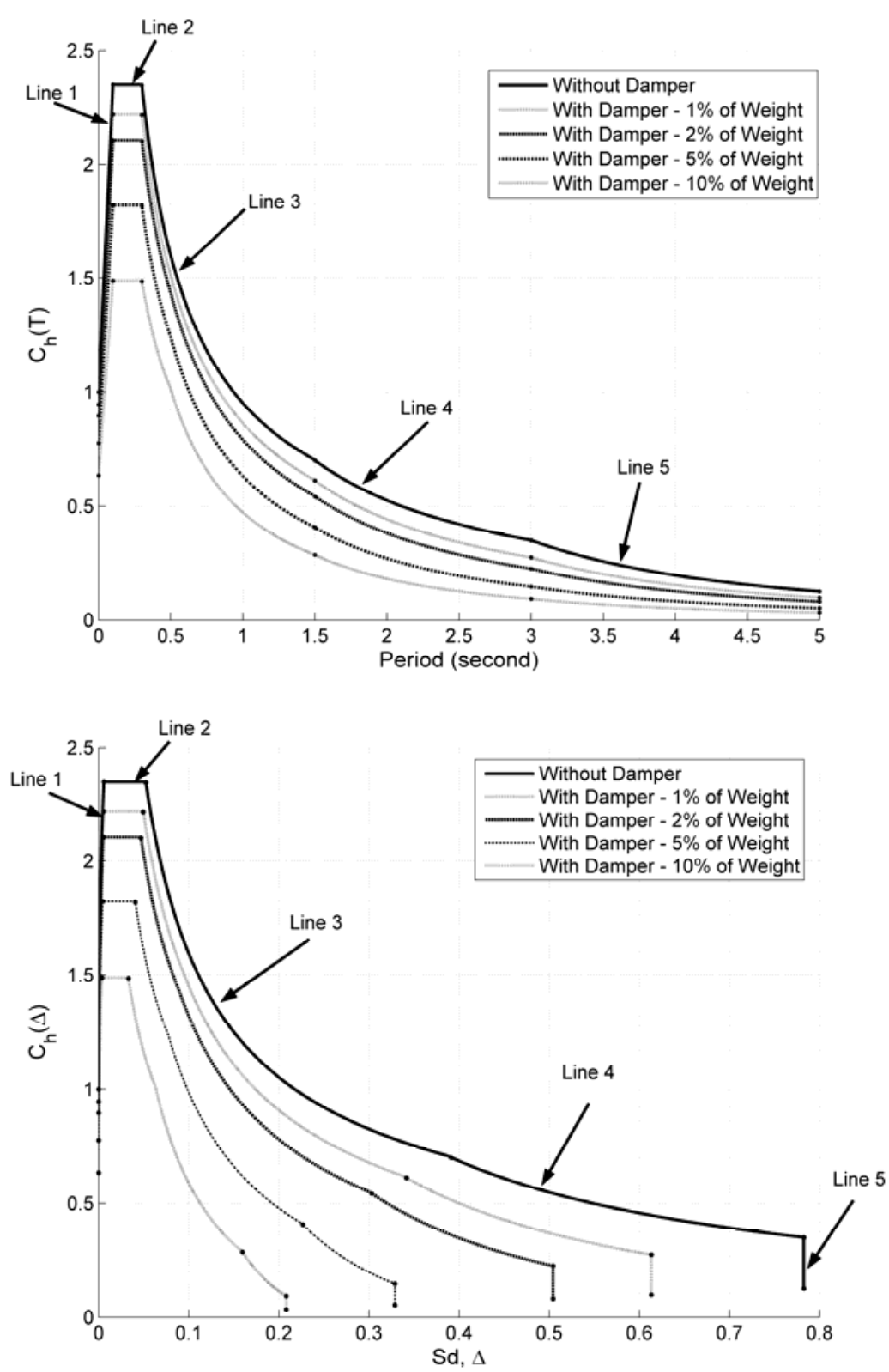

Figure 11: a) Design spectra based upon approximate damping reduction factor equations and spectral shape factors from NZ Structural Design Actions 1170.5; and b) Acceleration-coefficient $C_{h}(\Delta)$ vs displacement response spectra derived from the standard $\left(C_{h}(T)\right)$ design spectra. 


$$
\begin{aligned}
& C_{c}=C_{h} \frac{Z R}{B_{\varepsilon}} \\
& Z R=\frac{C_{c} B_{\varepsilon}}{C_{h}}
\end{aligned}
$$

When the appropriate reduction factor is used in place of the generic reduction factor, $B_{\varepsilon}$, the five branches of the spectrum thus become:

$$
C_{c}=\left(1+84.8 \sqrt{\frac{\Delta_{y}}{C_{c} g}}\right) \frac{Z R}{B_{a}}
$$

1.

$$
\begin{aligned}
& Z R=\frac{C_{c} B_{a}}{1+84.8 \sqrt{\frac{\Delta_{y}}{C_{c} g}}} \\
& C_{c}=2.35 \frac{Z R}{B_{a}}
\end{aligned}
$$

$$
Z R=0.426 C_{c} B_{a}
$$

$$
C_{c}=\frac{0.24}{B_{v}}\left(\frac{C_{c} g}{\Delta_{Y}}\right)^{0.375} Z R
$$

3.

4.

$$
Z R=4.167\left(\frac{\Delta_{y}}{g}\right)^{0.375} C_{c}^{0.625} B_{v}
$$

$$
C_{c}=0.167 \sqrt{\frac{C_{c} g}{\Delta_{y}}} \frac{Z R}{B_{v}}
$$

$$
Z R=6 \sqrt{\frac{\Delta_{y} C_{c}}{g}} B_{v}
$$

$$
C_{c}=0.08\left(\frac{C_{c} g}{\Delta_{y}}\right) \frac{Z R}{B_{d}}
$$

$$
Z R=12.5 \frac{\Delta_{y}}{g} B_{d}
$$

Note that reduction factor $B_{v}$ in Equations (18iii-iv) is a function of period, $T$, defined as $B_{v}=1+(8.72 T+1.44) \varepsilon$ where $\varepsilon$ is the non-dimensional damper capacity; substituting for the period using Equation (11) yields:

$$
\begin{aligned}
B_{v} & =1+\left(8.72\left(2 \pi \sqrt{\frac{\Delta_{y}}{C_{c} g}}\right)+1.44\right) \varepsilon \\
& =1+\left(54.8 \sqrt{\frac{\Delta_{y}}{C_{c} g}}+1.44\right) \varepsilon
\end{aligned}
$$

The derivation presented in Equations (6-19) enables easy incorporation of high force-to-volume dampers into design analyses through the simple procedure summarised in the following steps:

\section{STEP 1 - Design the structure without dampers}

STEP 2 - Fit a set of appropriate dampers and calculate the non-dimensional damper capacity contribution, $\varepsilon$.

STEP 3 - Assess the seismic performance capacity by taking the greater of:

3.1. $\quad(Z R)_{c}=\frac{C_{c}(1+5.8 \varepsilon)}{1+84.8 \sqrt{\frac{\Delta_{y}}{C_{c} g}}}$

3.2. $(Z R)_{c}=0.426 C_{c}(1+5.8 \varepsilon)$

3.3. $(Z R)_{c}=4.167\left(\frac{\Delta_{y}}{g}\right)^{0.375}\left(C_{c}\right)^{0.625}\left(1+\left(54.8 \sqrt{\frac{\Delta_{y}}{C_{c} g}}+1.44\right) \varepsilon\right)$

3.4. $(Z R)_{c}=6 \sqrt{\frac{C_{c} \Delta_{y}}{g}}\left(1+\left(54.8 \sqrt{\frac{\Delta_{y}}{C_{c} g}}+1.44\right) \varepsilon\right)$

3.5. $(Z R)_{c}=12.5 \frac{\Delta_{y}}{g}(1+27.6 \varepsilon)$

STEP 4 - Check the seismic performance capacity exceeds design demand. If $(Z R)_{c} \geq(Z R)_{d}$, the design is acceptable. If $(Z R)_{c}<(Z R)_{d}$, then either:

a) Increase degree of damping, $\varepsilon$

b) Increase base shear capacity, $C_{c}$

c) Permit limited, but an acceptable degree of inelasticity by replacing $\Delta_{y}$ with $\Delta=\mu \Delta_{y}$. Start with a ductility factor of, say, 1.5.

d) Use a combination of the above

And then return to Step 3 
The capacity spectrum generated by Equations (9i-v) is presented in Figure 11a, and can be transformed into the acceleration-displacement response spectra (ADRS) as detailed above. The five branches of the spectrum presented in Figure 11a are labelled as lines 1-5 and correspond to Equations (9i-v) respectively. The corresponding ADRS generated by Equations (12i-v) is presented in Figure 11b. It is important to note that the spectrum shape presented here are peak ground acceleration normalised, so these spectra require multiplication by the site PGA to obtain the appropriate spectrum for design purposes.

\section{CONCLUSIONS}

Dampers with force levels ranging from 100-400 kN have been experimentally developed that are sufficiently compact to fit into structural connections. Prestressing the lead is critical as it removes casting porosity, prevents the formation of a trailing void, and provides a beneficial compressive residual stress. The result is that the hysteresis loop is more optimally 'square' and the resistive forces generated and energy dissipated increase. To obtain the optimal hysteresis loop, a combination of careful device design and the application of prestress are important. Heating of the working material during high speed cyclic loading is an important consideration as the heating softens the lead and reduces the resistive force. Cyclic experiments have shown that these effects will not produce any notable effects for the 3-4 large cycles that are likely to occur during an earthquake.

Detailed trade-off analysis is used to develop simple design curves relating peak force levels to device design parameters. These trade-off curves allow force levels to be predicted for similar devices, and allow further devices to be easily designed for any structural application. An important trend seen in the experimental results is that the relationships between force and device parameters do not follow the extrusion models presented in other research. From this result, a new model of the device process is also briefly proposed and presented. Ongoing research has shown that these devices can be constructed with sufficiently small dimensions to be incorporated in the structural applications presented schematically within this paper [9].

Empirical equations to approximate damping reduction factors for a system with a lead extrusion damper are utilised based upon previous research but are now applied to New Zealand Structural Design Actions. The application to New Zealand Standards will act as an aid to creating a bridge to the profession, making this research more easily incorporated into structural design analyses. Overall, the compact high force/volume dampers presented are an important initial step towards a wide variety of novel applications and improved structural resilience.

\section{REFERENCES}

1 Robinson, W.H. and Greenbank, L.R., (1976). “An extrusion energy absorber suitable for the protection of structures during an earthquake". Earthquake Engineering and Structural Dynamics, 4(3):251-259.

2 Robinson, W.H. and Greenbank, L.R., (1975). "Properties of an extrusion energy absorber". Bulletin of the New Zealand National Society for Earthquake Engineering, 8(3):187-191.

3 Cousins, W. J. and Porritt, T. E. (1993). "Improvements to lead-extrusion damper technology," Bulletin of the New Zealand National Society for Earthquake Engineering, vol. 26, pp. 342-348.
4 Rodgers, GW, Denmead, C, Leach, N, Chase, JG, Mander, JB. (2006). "Spectral evaluation of high forcevolume lead dampers for structural response reduction," Proc. New Zealand Society for Earthquake Engineering Annual Conference, Napier, New Zealand, March 10-12.

5 Skinner, R.I., Robinson, W.H. and McVerry, G.H., (1993). "An Introduction to Seismic Isolation". John Wiley \& Sons, Chichester.

6 Pearsons, C. E. and Parkins, R. N. (1960). "The Extrusion of Metals," $2^{\text {nd }}$ Edition Revised, London: Chapman \& Hall Ltd, pp. 201-221.

7 Pekcan, G, Mander, J.B, and Chen, S.S. (1999). "Fundamental Considerations for the Design of Nonlinear Viscous Dampers," Earthquake Engineering and Structural Dynamics, vol. 28, pp. 1405-1425.

8 Standards New Zealand - Technical Committee BD-006-04, "NZS 1170.5 Supp 1:2004: Structural Design Actions - Part 5: Earthquake Actions - New Zealand Commentary"; Standards New Zealand, Wellington, New Zealand, 2004, Section C3.1, pp 17-28.

9 Solberg, KM, Bradley, BA, Rodgers, GW, Mander, JB, Dhakal, RP, and Chase, JG, (2007). "Multi-Level Seismic Performance Assessment of a Damage-protected Beamcolumn Joint with Internal Lead Dampers" Proc. New Zealand Society for Earthquake Engineering Annual Conference, Palmerston North, New Zealand, March 30April 1. 